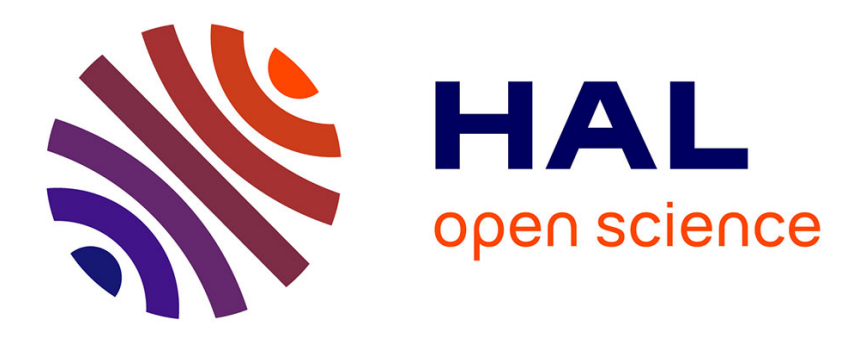

\title{
A 2.4 GHz active phase shifter architecture for smart antennas control
}

Florin Doru Hutu, David Cordeau, Jean-Marie Paillot

\section{To cite this version:}

Florin Doru Hutu, David Cordeau, Jean-Marie Paillot. A 2.4 GHz active phase shifter architecture for smart antennas control. IEEE MTT-S International Microwave Symposium, Jun 2009, Boston, United States. pp.821-824. hal-00683389

\section{HAL Id: hal-00683389 \\ https://hal.science/hal-00683389}

Submitted on 28 Mar 2012

HAL is a multi-disciplinary open access archive for the deposit and dissemination of scientific research documents, whether they are published or not. The documents may come from teaching and research institutions in France or abroad, or from public or private research centers.
L'archive ouverte pluridisciplinaire HAL, est destinée au dépôt et à la diffusion de documents scientifiques de niveau recherche, publiés ou non, émanant des établissements d'enseignement et de recherche français ou étrangers, des laboratoires publics ou privés. 


\title{
A $2.4 \mathrm{GHz}$ active phase shifter architecture for smart antennas control
}

\author{
Florin HUTU, David CORDEAU and Jean-Marie PAILLOT
}

\author{
LAII, EA 1219, IUT Angoulême, 4 av. de Varsovie, 16021 Angoulême Cedex, France \\ jmpailloteiutang.univ-poitiers.fr
}

\begin{abstract}
This paper aims at developing the bases of a smart radio-communication prototype, able to modify the radiation patterns, both on emission and reception, according to the needs of the communication. Smart antenna is a well-known concept, allowing to increase the quality of the transmission and spectral efficiency. To control the radiation patterns, algorithms used in smart antenna techniques require precise amplitudes and phases generation. In this paper, vector modulators working as active phase shifters will be used in order to synthesize such amplitudes and phases. A theoretical analysis will be performed in order to quantify the impact of vector modulators mismatches on the synthesized amplitudes and phases. Furthermore, measurements of radiation patterns generated by a prototype at $2.4 \mathbf{G H z}$ will be presented.
\end{abstract}

Index Terms-smart antenna systems, active phase shifters, beam forming techniques.

\section{INTRODUCTION}

The Internet development has created a need for access data and multimedia that is much more than a simple voice communication. Moreover, it is expected to access these data anywhere and on any device. Communication systems having beamforming technology has already proven their efficiency by increasing the spectral efficiency and reducing the multi path fading, the BER and the co-channel interferences.

This is possible by electronically adjusting the radiation pattern of the antenna so that a high gain is presented in the signal of interest direction and null gains in the interference signals directions. To do so, signals with the same frequency but different phases and amplitudes must be generated.

Few solutions, with phase-shifters or phase-shifterless, are proposed to achieve this electronic modification of the radiation pattern. Indeed, passive phase shifters can be used to perform the phase control. Unfortunately their losses decrease the overall system performances. Others solutions like Rotman lenses or Butler matrices can also be used especially for switched beam antenna arrays.

On the other hand, an active solution presented by J. J. Lynch and R. A. York [1] uses coupled oscillator array to control the relative phases though adjustment of oscillators' natural frequencies. Since these seminal works, only few papers have presented new investigations and improvements, in particular concerning side lobes reduction [2].

In this context, this paper presents an original architecture, based on vector modulators used as active phase shifters, to perform beamforming by controlling the phases and amplitudes of the excitation signals.

After a brief review of smart antenna principle presented in section II, the original active phase shifter architecture is discussed in section III. In particular, the influence of the vector modulator mismatches on the synthesized phase and amplitude will be studied. Finally, section IV shows the measurements of radiation patterns generated by the prototype at $2.4 \mathrm{GHz}$.

\section{SMart ANTEnNa Principle}

A smart antenna is made of an array of individual radiative elements (elementary antennas), which are placed in a particular configuration (linear, circular or matrix). It is also made of an RF front-end which, among other functions, must change the amplitudes and phases of the signals associated to each elementary antenna. The RF front-end is driven by a DSP which dynamically compute the amplitudes and phases values in order to maximize the performances of the communication system.

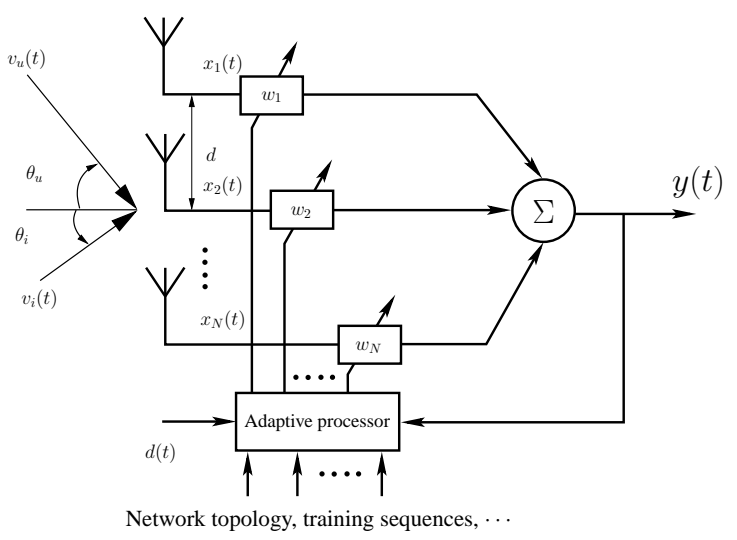

Fig. 1. General diagram of smart antenna system

By gathering the elementary antennas in such arrays and by changing the characteristics of the signals associated to each element, the array can present different gains according to the direction and due to the property of the reciprocity of the antennas, the radiation patterns are identical both on emission and reception.

Let us consider the case of a uniform linear array of $N$ elementary antennas placed at the same distance $d$ between 
them as in Fig. 1. In order to study the behavior of this configuration, let us suppose that the signal provided by each elementary antenna is multiplied by a complex coefficient $w_{m}=A_{m} e^{j \phi_{m}}$; where $m=1, \cdots, N$.

The signal of interest $v_{u}(t)$ and the interference signal $v_{i}(t)$ will generate an output $y(t)$ as follows:

$$
y(t)=\mathbf{w}^{H}\left[v_{u}(t) \cdot \mathbf{v}_{u}+v_{i}(t) \cdot \mathbf{v}_{i}\right]
$$

where $\mathbf{w}^{H}$ is the Hermitian conjugate of the vector containing the complex coefficients $w_{m}$ and $\mathbf{v}_{u}, \mathbf{v}_{i}$ are two vectors depending on the interest $\theta_{u}$ and interference $\theta_{i}$ directions, so that:

$$
\mathbf{v}_{u}=\left[\begin{array}{c}
1 \\
e^{\jmath k_{0} d \cos \theta_{u}} \\
e^{\jmath 2 k_{0} d \cos \theta_{u}} \\
\vdots \\
e^{\jmath(N-1) k_{0} d \cos \theta_{u}}
\end{array}\right] \mathbf{v}_{i}=\left[\begin{array}{c}
1 \\
e^{\jmath k_{0} d \cos \theta_{i}} \\
e^{\jmath 2 k_{0} d \cos \theta_{i}} \\
\vdots \\
e^{\jmath(N-1) k_{0} d \cos \theta_{i}}
\end{array}\right]
$$

with $k_{0}=\frac{2 \pi}{\lambda}$, the propagation constant.

Considering $d(t)$, as version of the $v_{u}(t)$ signal locally generated, an error signal $\varepsilon(t)$ can be defined as follows:

$$
\varepsilon(t)=y(t)-d(t)
$$

The complex weights $\mathbf{w}$ minimizing the quadratic error $|\overline{\varepsilon(t)}|^{2}$ are found by using a gradient approach and the Lagrange multipliers method. The following algorithm is obtained [3]:

$$
\begin{aligned}
& \text { do } \\
& \text { while } \quad \mathbf{w}[n+1]=\mathbf{w}[n]+\mu \cdot \varepsilon[n] \cdot \mathbf{x}[n]
\end{aligned}
$$

where $\mu$ represents a step size controlling the convergence of the algorithm, $\varepsilon_{0}$ the maximum allowed level of error and $\mathbf{x}[n]=\left[x_{1}, x_{2}, \cdots x_{N}\right]$ represents the vector containing the samples of the inputs $x_{1}(t) \cdots x_{N}(t)$.

This algorithm is widely used due to its simplicity and its low computational complexity. For example, let us consider an array of $N=4$ identical equidistant antennas $\left(d=\frac{\lambda}{2}, f_{0}=\right.$ $2.4 \mathrm{GHz}$ ). Furthermore, let us suppose that the direction of the desired signal is $\theta_{p}=30^{\circ}$ and the direction of the interference signal is $\theta_{i}=-15^{\circ}$. In this particular case, using the previous algorithm, the obtained amplitudes and phases, computed as the modules and the phases of the complex weights $\mathbf{w}$, are the following :

\begin{tabular}{|c||c|c|c|c|}
\hline$A_{m}$ & 0.21 & 0.29 & 0.29 & 0.21 \\
\hline$\phi_{m}$ & $10.51^{\circ}$ & $-110.4^{\circ}$ & $176.3^{\circ}$ & $55.3^{\circ}$ \\
\hline
\end{tabular}

TABLE I

AMPLITUDES AND PHASES NECESSARY FOR 4 ANTENNAS IN THE

$$
\theta_{u}=30^{\circ} \text { AND } \theta_{i}=-15^{\circ} \text { SCENARIO }
$$

Using these amplitudes and phases, the total antenna array gain is:

$$
G_{\text {tot }}(\theta, \phi)=G_{r e f}(\theta, \phi) \cdot \sum_{m=1}^{N} A_{m} e^{\jmath\left(\phi_{m}+k_{0} d \cos \theta\right)}
$$

where $G_{r e f}(\theta, \phi)$ represents the gain of an elementary antenna.

To electronically modify the radiation patterns, several techniques have been used as mentioned below.

Recently, Ali Hajimiri and his work group proposed a solution using a polyphase VCO, able to generate the LO frequency with $N$ phases. In this architecture, all the $N$ phases are conveyed to each antenna via a distribution network. Phase selectors ensure the required phase to each element [4]. In this case, the phase variations are discrete which does not constitute a problem as long as the discrimination steps is adequate to the application. Nevertheless, the distribution network of $N$ phases constitutes here a real issue since each line must be forwarded to the phase selector in a symmetrical way.

Another solution, initiated by R. A. York is based on the synchronization of an array of oscillators having free running frequencies with a weak dispersion. In [1], the synchronization of non linear coupled oscillators arrays is proved. Moreover, according to the coupling strength and to free-running frequencies, phase variations can be provided. Recent works [2] present how the phase variation can be supplied by changing only the free-running frequencies of all coupled oscillators in the array. Despite the fact that a continuous phase variation can be obtained, this technique seems to be relatively complex to implement and the theoretical maximum relative phase is equal to $\pm 90^{\circ}$.

Let us note that the previous techniques allow to control only the phases of the signals applied to each elementary antenna which is not sufficient for beamforming techniques as shown in Table I.

\section{Active Phase Shifter Architecture}

The original architecture presented in this paper uses vector modulators, as active phase shifters, to achieve the required amplitudes and phases applied to each elementary antennas. Very few articles [5], [6], [7] were published on this solution which, however seems to be a promising one.

In a beamforming system, the precision of the radiation pattern depends on the accuracy of the synthesized amplitudes and phases applied to each elementary antenna.

In order to quantify the impact of vector modulators mismatches on the synthesized amplitudes and phases, let us consider a common vector modulator structure (Fig. 2). As shown on this figure the basic function blocks are a filter for quadrature signals generation, two single to differential buffers, two Gilbert cell multipliers and an output stage.

If all the elements in this structure have an ideal behavior and if the single to differential buffers and the output buffer compensate the attenuations produced by the quadrature filter 


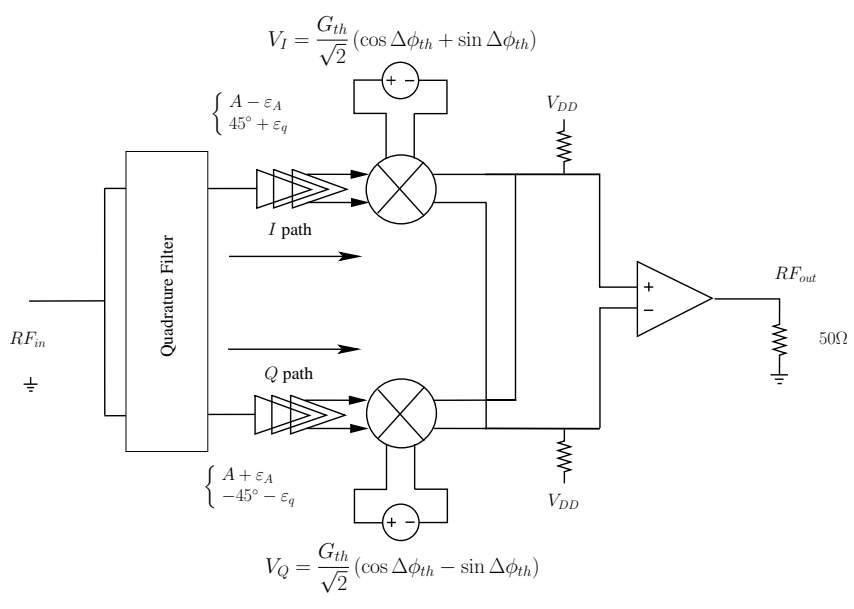

Fig. 2. A common vector modulator based active phased shifter

and Gilbert cells, the gain of the active phase shifter is $G_{t h}$ and the synthesized phase shift is $\Delta \phi_{t h}$.

Let us define $\varepsilon_{A}$ and $\varepsilon_{q}$ the amplitude mismatch and phase imbalance respectively in the $I$ and $Q$ path of the active phase shifter.

Then, the relative gain error $\varepsilon_{r e l, G}$ and the absolute phase error $\varepsilon_{\Delta \phi}$ can be expressed as follows :

$$
\left\{\begin{array}{l}
\varepsilon_{r e l, G} \triangleq \frac{G_{N}-G_{t h}}{\left|G_{t h}\right|} \cdot 100 \\
\varepsilon_{\Delta \phi} \triangleq\left|\Delta \phi_{N}-\Delta \phi_{t h}\right|
\end{array}\right.
$$

where $G_{N}$ and $\Delta \phi_{N}$ are the obtained gains and phase shifts in the presence of mismatch.

The relative gain error $\left.\varepsilon_{r e l, G}\right|_{\varepsilon_{A}}$ and the absolute phase error $\left.\varepsilon_{\Delta \phi}\right|_{\varepsilon_{A}}$, due to an $\varepsilon_{A}$ amplitude mismatch can be then written as follows:

$$
\left\{\begin{array}{l}
\left.\varepsilon_{r e l, G}\right|_{\varepsilon_{A}}=\left(-1+\sqrt{1+\left(\frac{\varepsilon_{A}}{A}\right)^{2}}\right) \cdot 100 \\
\left.\varepsilon_{\Delta \phi}\right|_{\varepsilon_{A}}=\left|-\arctan \frac{\varepsilon_{A}}{A}\right|
\end{array}\right.
$$

where $A$ represents the filters output amplitudes without mismatch.

Fig. 3. shows both relative gain error $\varepsilon_{r e l, G}$ and absolute phase error $\varepsilon_{\Delta \phi}$ versus $\varepsilon_{A}$ amplitude mismatch. It can be observed that for an amplitude mismatch less than $A / 4$, the relative gain error is less than $3 \%$ and the absolute phase error less than $15^{\circ}$.

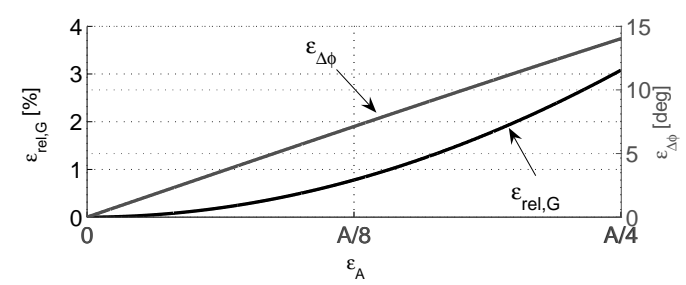

Fig. 3. Relative gain error $\varepsilon_{r e l, G}$ and absolute phase error $\varepsilon_{\Delta \phi}$ due to amplitude mismatch $\varepsilon_{A}$
The relative gain error $\left.\varepsilon_{r e l, G}\right|_{\varepsilon_{q}}$ and the absolute phase error $\left.\varepsilon_{\Delta \phi}\right|_{\varepsilon_{q}}$, due to a $\varepsilon_{q}$ quadrature mismatch can be written as follows:

$$
\left\{\begin{array}{l}
\left.\varepsilon_{r e l, G}\right|_{\varepsilon_{q}}=\left(-1+0.5 \cdot \sqrt{M^{2}+N^{2}}\right) \cdot 100 \\
\left.\varepsilon_{\Delta \phi}\right|_{\varepsilon_{q}}=\left|\arctan \frac{N}{M}-\Delta \phi_{t h}\right|
\end{array}\right.
$$

with:

$$
M=\operatorname{det}\left\{\left[\begin{array}{cc}
\cos \varepsilon_{q}-\sin \varepsilon_{q} & \sin \varepsilon_{q}-\cos \varepsilon_{q} \\
\cos \Delta \phi_{t h}-\sin \Delta \phi_{t h} & \cos \Delta \phi_{t h}+\sin \Delta \phi_{t h}
\end{array}\right]\right\}
$$

and

$$
N=\operatorname{det}\left\{\left[\begin{array}{cc}
\sin \varepsilon_{q}+\cos \varepsilon_{q} & \sin \varepsilon_{q}+\cos \varepsilon_{q} \\
\cos \Delta \phi_{t h}-\sin \Delta \phi_{t h} & \cos \Delta \phi_{t h}+\sin \Delta \phi_{t h}
\end{array}\right]\right\}
$$

By using equations (8), Fig. 4. and Fig. 5. can be plotted. In Fig. 4., the dark regions represents areas in the $\left(\varepsilon_{q}, \Delta \phi_{t h}\right)$ plane where the relative gain error is greater than $10 \%$. One can say that a relative gain error less than $10 \%$ is obtained for quadrature errors less than $5^{\circ}$ for all synthesized phase shifts.

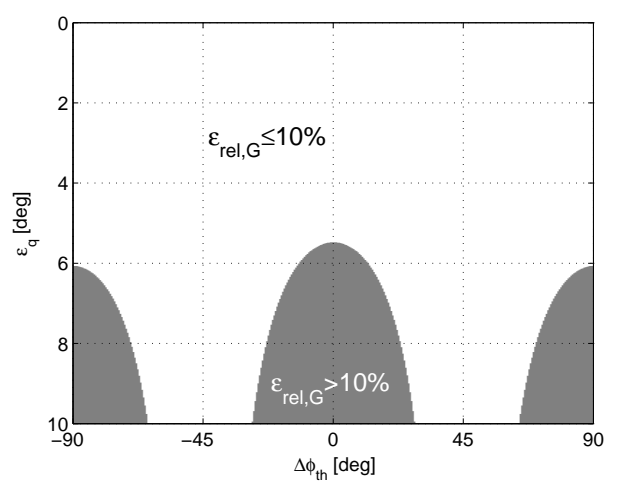

Fig. 4. Relative gain error $\varepsilon_{r e l, G}$ due to quadrature error $\varepsilon_{q}$

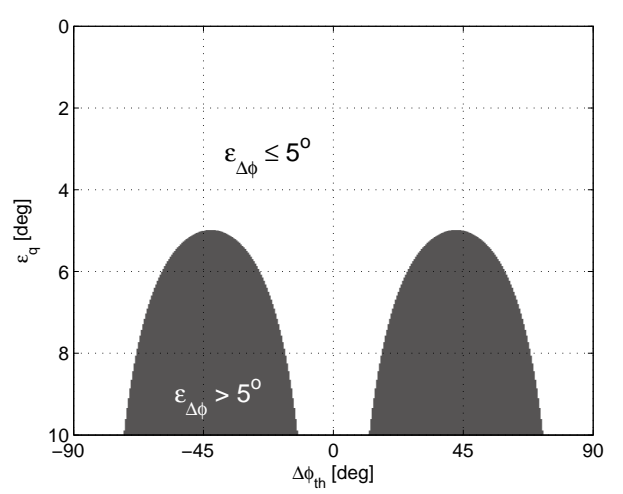

Fig. 5. Absolute phase shift error $\varepsilon_{\Delta \phi}$ due to quadrature error $\varepsilon_{q}$

In Fig. 5., the dark regions correspond to pairs $\left(\varepsilon_{q}, \Delta \phi_{t h}\right)$ where the absolute phase shift error is greater than $5^{\circ}$. One 
can say that a absolute phase error less than $5^{\circ}$ is obtained for quadrature errors less than $5^{\circ}$ for all synthesized phase shifts.

The Gilbert cell multiplier do not introduce errors while the input levels are such that the multiplier works in the linear region of operation.

Concerning the transmission performances, the influence of perturbations on the command signals $V_{I}$ and $V_{Q}$ was presented in [8]. System simulations proved that a $C_{\min } / N>$ $40 \mathrm{~dB}^{1}$ ratio does not affect performances in terms of EVM and BER.

By taking into account all these constraints, the design of a prototype using active phase shifters become possible. This prototype is the first step to a future integrated system.

\section{Measurement Results}

A prototype with four "patch" antennas, using discrete components was implemented. The elementary command circuit uses an AD 8394 vector modulator and is followed by an ADL 5330 Voltage Gain Amplifier. Input and output 1:1 transmission line transformers allow the single to differential mode transitions. Four such circuits are driven by an RF signal equally split by a distribution tree. The command circuit output are connected to "patch" antennas. The distribution tree and the patch antennas are implemented on a epoxydic substrate with $\varepsilon_{r}=4.6 ; \tan \delta=10^{-2}$ and conductive layer height $h=0.35 \mu \mathrm{m}$.

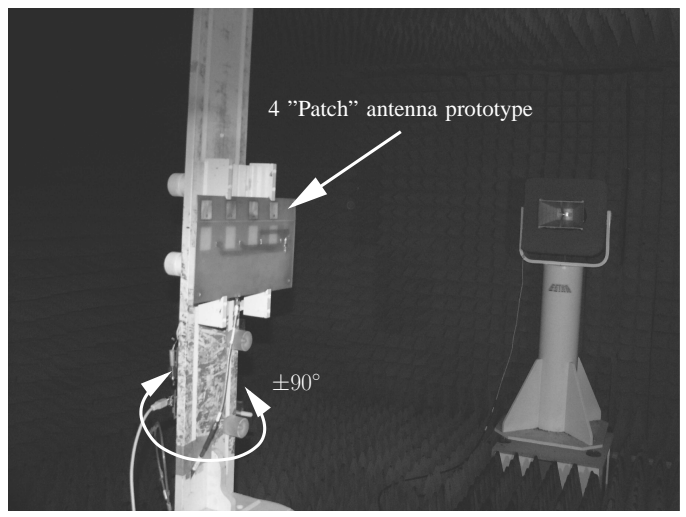

Fig. 6. The 4 "patch" antennas prototype in anechoic chamber

Using this prototype, measurements in anechoic chamber (Fig. 6) were performed. $V_{I}$ an $V_{Q}$ DC voltages corresponding to amplitudes and phases given in Table I were applied. An RF signal @ $2.4 \mathrm{GHz}$ and $-10 \mathrm{dBm}$ power, provided by a Wiltron 360, $40 \mathrm{GHz}$ Network Analyzer was also applied to the prototype.

Fig. 7. shows the theoretical and measured radiation patterns. As expected, the measured radiation pattern has a maximum in the $31^{\circ}$ direction and a minimum of radiation in $-15^{\circ}$ direction. Furthermore, let us note that a very good agreement can be found between experimental and theoretical results. The small difference between the two radiation patterns is due to

\footnotetext{
${ }^{1} C_{\min }$ correspond to the minimum value of the command voltages $V_{I}$ and $V_{Q}$
}

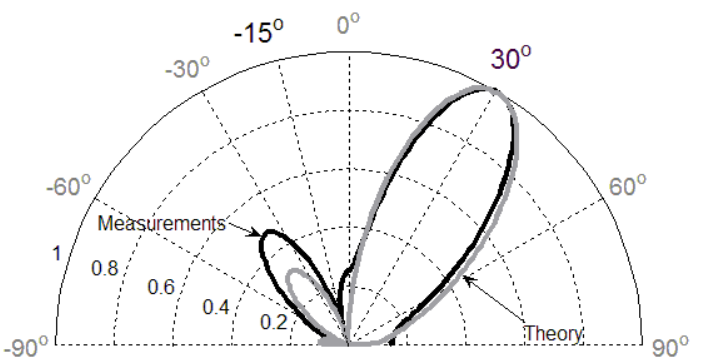

Fig. 7. Measured and theoretical normalized radiation patterns

the fact that elementary antennas do not have exactly the same behavior.

\section{CONCLUSiON}

A novel approach for smart antenna command was presented. Electronic beamforming using vector modulators as active phase shifters presents a lot of advantages, in particular an improved SNR during transmission, and a better transmission of the transmitters' output power. In this paper, a theoretical analysis was performed in order to quantify the impact of vector modulator mismatches on the synthesized amplitudes and phases applied on each elementary antennas. By taking into account these non ideal behaviors, a $2.4 \mathrm{GHz}$ prototype with 4 "patch" antennas was implemented and characterized in anechoic chamber. A very good agreement was found between the measurements and theoretical results.

\section{ACKNOWLEDGMENT}

The authors thanks the "Poitou Charentes" region council for their financial support of this work. Also, they would like to thank M. David CARSENAT, Cyril DECROZE and Moctar MOUHAMADOU from Xlim research institute, Limoges, France for helpful discussions and the contribution to the radiation pattern measurements.

\section{REFERENCES}

[1] J. J. Lynch and R. A. York, "Synchronization of Oscillators Coupled Through Narrow-Band Networks," IEEE Transactions on Microwave Theory and Techniques, vol. 49, no. 2, pp. 237-249, February 2001.

[2] T. Heath, "Simultaneous Beam Steering and Null Formation With Coupled, Nonlinear Oscillator Arrays," IEEE Transactions on Antennas and Propagation, vol. 53, no. 6, pp. 2031 - 2035, June 2005.

[3] I. Frost, O.L., "An algorithm for linearly constrained adaptive array processing," Proceedings of the IEEE, vol. 60, no. 8, pp. 926 - 935, Aug. 1972.

[4] A. Natarajan, A. Komijani, and A. Hajimiri, "A fully integrated 24-GHz phased-array transmitter in CMOS," Solid-State Circuits, IEEE Journal of, vol. 40, no. 12, pp. 2502-2514, Dec. 2005.

[5] S. Gueorguiev, S. Lindfors, and T. Larsen, "A 5.2 GHz CMOS I/Q Modulator With Integrated Phase Shifter for Beamforming," IEEE Journal of Solid-State Circuits, vol. 42, no. 9, pp. 1953 - 1962, September 2007.

[6] K.-J. Koh and G. M. Rebeiz, "0.13- $\mu$ m CMOS Phase Shifters for X-, $\mathrm{Ku}-$, and K-Band Phased Arrays," IEEE Journal of Solid-State Circuits, vol. 42, no. 11, pp. 2535 - 2546, november 2007.

[7] _ "An X- and Ku-Band 8-Element Phased-Array Receiver in 0.18$\mu m$ SiGe BiCMOS Technology," IEEE Journal of Solid-State Circuits, vol. 43, no. 6, pp. 1360 - 1371, June 2008.

[8] N. Tohmé, J.-M. Paillot, D. Cordeau, S. Cauet, Y. Mahé, and P. Ribardière, "A $2.4 \mathrm{GHz}$ 1-Dimensional Array Antenna driven by Vector Modulators," in IEEE MTT-S International Microwave Symposium, 2008. 